\title{
Optimal Indexing of the Vertices of Graphs*
}

\author{
By Carl H. FitzGerald
}

\begin{abstract}
The incidence matrices of various graphs are considered. By reordering the points, the bandwidth can be changed. In the cases of rectangular grids in the plane or cubic grids in three dimensions, the exact, minimum values of the bandwidth are determined.

In certain numerical analysis problems, it is of interest to index the vertices of a graph in such a way that the matrix used to represent an associated system of linear equations is as close to diagonal as possible or, equivalently, to index the vertices of a graph in a way that minimizes the width of the band of nonzero terms in the incidence matrix for that graph. The purpose of this note is to present a few methods of determining the minimum possible width in some cases that arise in the numerical solution of Laplace's equation. Of particular interest are the first proof of Theorem 2 which solves the problem for the common square grid and Theorem 3 which answers the problem for the cubic grid.
\end{abstract}

As depicted by the solid lines of Fig. 1, let $\mathcal{G}_{n}$ be the graph consisting of vertices being points $(i, j)$ where $i$ and $j$ are integers such that $|i|+|j| \leqslant n$ and of edges being the straight line segments joining vertices that are exactly one unit apart. The vertices are to be indexed with distinct integers. Let $\delta$ be the minimum over all indexing of the maximum difference between the numbers assigned adjacent vertices.

TheOREM 1. $\delta=n+1$ for $n=1,2, \ldots$.

Proof. Counting down the dotted diagonal at the upper right of Fig. 1, there are $n+1$ vertices $(i, j)$ of $\mathcal{G}_{n}$ such that $i+j=n$. Skipping to the third diagonal, there are another $n+1$ such that $i+j=n-2$; and so forth until there are $n+1$ such

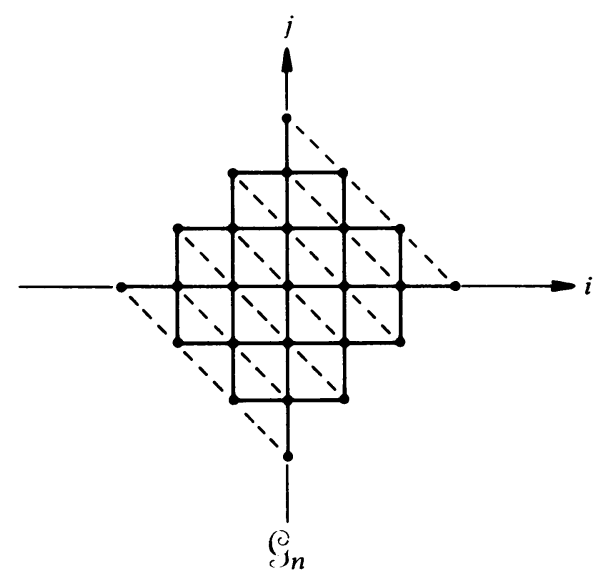

FIGURE 1

Received June 11, 1973.

AMS (MOS) subject classifications (1970). Primary 05C35; Secondary 65N 10, 05 B20.

Key words and phrases. Bandwidth, incidence matrix, grids.

* This research was supported in part by the National Science Foundation Grant NSF GP32156.

Copyright $\odot 1974$, American Mathematical Society 
that $i+j=-n$. There are $(n+1)^{2}$ points which are counted by this procedure. Counting down the diagonals omitted before, there are $n$ points on each of $n$ skipped diagonals. Thus, there are $(n+1)^{2}+n^{2}=2 n^{2}+2 n+1$ vertices in $\mathcal{G}_{n}$. For any indexing, it takes no more than $2 n$ edges to go from the vertex to which 1 is assigned to that which $2 n^{2}+2 n+1$ is assigned. Thus, $2 n \cdot \delta \geqslant\left(2 n^{2}+2 n+1\right)$ -1 ; hence $\delta \geqslant n+1$.

It is easy to see this estimate is sharp by indexing the vertices consecutively down the diagonal for which $i+j=n$, then down the diagonal for which $i+j=n-1$, etc.

Remark. If the dotted line segments of Fig. 1 are included in $\mathcal{S}_{n}$, the value for the corresponding $\delta$ is unchanged. This modified graph is homeomorphic to a graph as depicted in Fig. 2 in which each interior point is the center of an equilateral hexagon.

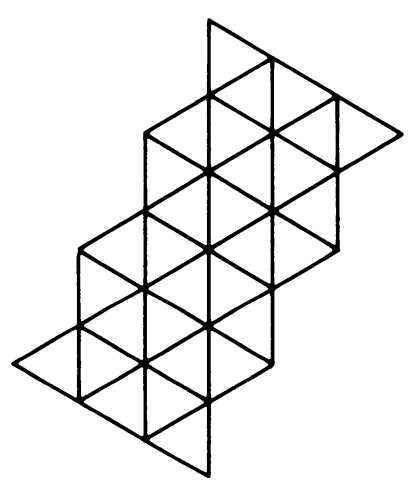

FIGURE 2

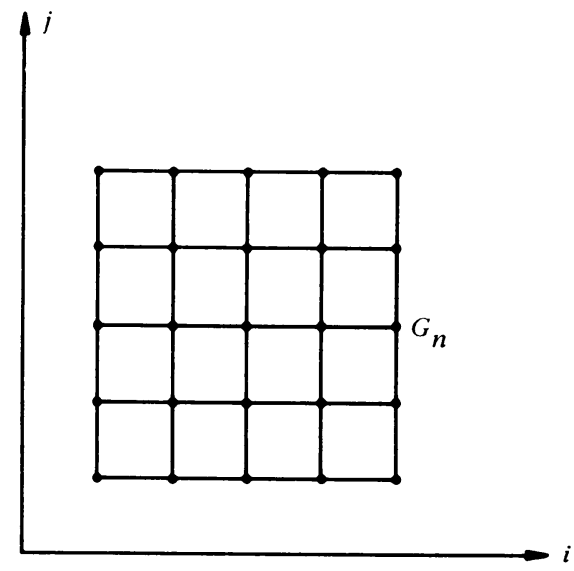

FIGURE 3

Consider the square grid $G_{n}$ as depicted by the solid line segments of Fig. 3. The vertices are the points $(i, j)$ such that $i$ and $j$ are integers, $1 \leqslant i, j \leqslant n$, and the edges are the straight line segments joining vertices which are exactly one unit apart. Let $\Delta$ be the minimum over all indexings of the maximum difference between the integers assigned adjacent vertices.

THEOREM 2. $\Delta=n$ for $n=2,3, \ldots$.

(This fact is contained in a theorem in the reference.)

First Proof.

LEMMA. Let $G_{n}$ be indexed. There exists an integer $q$ such that either every column or every row of $G_{n}$ contains adjacent points assigned integers $r$ and such that $r \leqslant q \leqslant s$.

Proof. Let $S_{i}=$ integers assigned vertices in the $i$ th column $\}$ for $1 \leqslant i \leqslant n$. For each $i$, consider the smallest integer in $S_{i}$; among these numbers there is a largest integer $q$. Let $i_{1}$ be the index of the $S_{i}$ to which $q$ belongs.

If, for a particular $i, S_{i}$ has an integer larger than $q$, there is also a point assigned an integer less than or equal to $q$ by choice of $q$; and thus there is a pair of adjacent points in the $i$ th column having assigned numbers $r$ and $s$ such that $r \leqslant q<s$. 
Hence, if, for each $i=1,2, \ldots, n, S_{i}$ has an integer larger than $q$, then every column has a pair as indicated by the lemma.

On the other hand, suppose, for some $i_{2}, S_{i_{2}}$ does not have an integer greater than $q$. Since $q$ belongs to $S_{i_{1}}$ and $i_{1}$ is not $i_{2}, q$ is greater than every number in $S_{i_{2}}$ and less than or equal to every number in $S_{i_{1}}$. Hence, in each row, there is a pair of distinct points assigned numbers $r$ and $s$ such that $r \leqslant q \leqslant s$; and, consequently, a pair of adjacent points in each column having such assigned numbers.

Proof of Theorem. Consider the $n$ pairs of integers the lemma says exist. Let $r^{\prime}$ be the smallest of these integers, and $s^{\prime}$ be the other member of the pair. Then $r^{\prime} \leqslant q-(n-1)$ and $q \leqslant s^{\prime}$ with equality holding in at most one of these inequalities since each integer occurs at most once in the indexing. Hence $s^{\prime}-r^{\prime} \geqslant n$, i.e., $\Delta \geqslant n$.

That $\Delta \leqslant n$ follows by considering the indexing starting at the lowest row of $G_{n}$ and numbering across in the same direction on successive rows.

Remarks. Consider the grids of the types mentioned in Theorems 1 and 2 for larger and approximately equal numbers of points. The band width of the incidence matrix for the graph mentioned in Theorem 1 is about $1 / \sqrt{2}$ times that for the graph of Theorem 2.

Clearly, Theorem 2 is sharp for $n \times m$ rectangular grids for which $m \geqslant n$. Furthermore, if the diagonals going across the $n \times m$ grid in one direction are included, the estimate of Theorem 2 remains sharp. An optimal indexing is obtained by numbering down successive diagonals starting at a corner not on a diagonal. Such a grid is homeomorphic to a graph as depicted in Fig. 4 in which each interior point is the center of an equilateral hexagon.

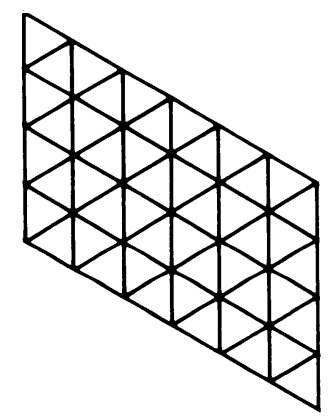

FIGURE 4

Most of the second proof is concerned with the following question: How can the vertices of the grid be indexed so that those labelled $1,2, \ldots, N$ are adjacent to the minimal number of other vertices? Clearly, it is desirable that the vertices labelled $1,2, \ldots, N$ be close together and in a corner. Thus, the answer found is independent of $N$. Roughly speaking, the proof shows that one scheme is to index starting in a corner and progress through the points closest to that corner.

The value of $\delta$ is then easily determined. A lower bound is found by considering the preceding question for $N$ approximately $\frac{1}{2} n^{2}$. The number of vertices adjacent to one of those labelled $1,2, \ldots, N$, but not indexed with one of those numbers, must be less than $\delta$. On the other hand, the indexing previously described has no jumps between adjacent vertices larger than this lower bound. 
Second Proof of Theorem 2. For any subset $T$ of the vertices of $G_{n}$, let $\sigma(T)$ be the number of vertices of $G_{n}$ not in $T$, but adjacent to points of $T$. Consider a subset $S$ of the vertices of $G_{n}$ with more than $n(n-1) / 2$ points, but less than $n(n+1) / 2$ points. It will be shown that $\sigma(S) \geqslant n$.

For each $i, 0 \leqslant i \leqslant n+1$, let $N_{i}$ be the number of points of $S$ in the $i$ th column. (Note $N_{0}=N_{n+1}=0$.) Let $S^{*}$ be $\left\{(i, j):(i, j)\right.$ belongs to $G_{n}$ and $\left.1 \leqslant j \leqslant N_{i}\right\}$.

Let $M_{i}$ be the number of points in the $i$ th column of $G_{n}$ that are adjacent to points of $S^{*}$, but not in $S^{*}$. Then, for $1 \leqslant i \leqslant n$,

$$
\begin{aligned}
M_{i} & =\max \left(N_{i+1}, N_{i-1}\right)-N_{i} \quad \text { if } N_{i+1} \text { or } N_{i-1}>N_{i}, \\
& =1 \quad \text { if } N_{i+1} \leqslant N_{i} \text { and } N_{i-1} \leqslant N_{i} \text { and } N_{i}<n, \\
& =0 \text { if } N_{i}=n .
\end{aligned}
$$

Clearly, $\sigma\left(S^{*}\right)=\sum_{i=1}^{n} M_{i}$.

By considering each column separately, it is easily verified that $\sigma(S) \geqslant \sigma\left(S^{*}\right)$.

Similarly, $S^{* *}$ is defined to be the subset of $G_{n}$ having the same number of vertices in each row as $S^{*}$ does and these points being the first vertices in the respective row. Then $\sigma(S) \geqslant \sigma\left(S^{*}\right) \geqslant \sigma\left(S^{* *}\right)$. If $(i, j)$ is in $S^{* *}$, then every point $\left(i^{\prime}, j^{\prime}\right)$ for which $1 \leqslant i^{\prime} \leqslant i$ and $1 \leqslant j^{\prime} \leqslant j$ is in $S^{* *}$.

If $S^{* *}$ contained no points $(i, j)$ for which $i+j=n+1$, then $S^{* *}$ would be contained in $\{(i, j): 1 \leqslant i, j \leqslant n$ and $i+j<n+1\}$. But the last set has only $n(n-1) / 2$ points, which is less than the number of points in $S$. Hence $S^{* *}$ contains at least one point $(i, j)$ for which $i+j=n+1$. If $S^{* *}$ contained all the vertices $(i, j)$ of $G_{n}$ such that $i+j=n+1$, then $S^{* *}$ would contain $\{(i, j): 1 \leqslant i \leqslant n$ and $1 \leqslant j \leqslant n$ and $i+j \leqslant n+1\}$. The last set has $n(n+1) / 2$ points which is more than the number of points in $S$. Thus, there exists a point $(I, J)$ of $G_{n}$ such that $I+J=n+1$ and either $(I-1, J+1)$ or $(I+1, J-1)$ is a point of $G_{n}$ not in $S^{* *}$.

Let $s$ be $\{(i, j): 1 \leqslant i \leqslant I$ and $1 \leqslant j \leqslant J\}$. Adjoin to $s$ the $J+1$ row of $S^{* *}$ making $s^{\prime}$. If $J+1<n$, there are two possibilities:

Case 1. $\sigma\left(s^{\prime}\right)=\sigma(s)$, if there are less than $I$ elements in the $J+1$ row.

Case 2. $\sigma\left(s^{\prime}\right)=\sigma(s)+1$, if there are exactly $I$ elements in the $J+1$ row.

By choice of $(I, J)$, there cannot be more than $I$ elements in the $J+1$ row of $S^{* *}$. For each successive row up to the $n$ th, similar cases apply.

As columns of $S^{* *}$ are adjoined, the value of $\sigma$ behaves in a similar way as in the adjoining of rows. (By choice of $(I, J)$, either the $J+1$ row or the $I+1$ column is not in Case 2 ; thus $(I+1, J+1)$ is not counted more than once.)

When the $n$th column of $S^{* *}$ and the $n$th row of $S^{* *}$ are adjoined, $\sigma$ may be decreased. Because of the creations of Case 2 when other columns and rows are adjoined, the net result is a net loss of at most 1 . Hence $\sigma(S) \geqslant I+J-1=n$.

Consider any indexing of the vertices of $G_{n}$. Let $S$ be the set of vertices labeled $1,2, \ldots, n(n-1) / 2+1$. Since $\sigma(S) \geqslant n$, there exists a pair of adjacent vertices of $G_{n}$, one in $S$ and one out, such that the indices differ by at least $n$. Thus $\Delta \geqslant n$.

As before, $\Delta \leqslant n$ is clear by an example indexing.

Using the method of the second proof of Theorem 2, it is easy to prove the following more general observation:

Corollaky. For $1 \leqslant l \leqslant n^{2}$, let $T_{l}$ be the subset of $G_{n}$ obtained by including points 
on the diagonals $i+j$ being constant starting with the diagonal $i+j=2$ and moving down successive diagonals until l points have been obtained. If $S$ is a subset of $G_{n}$ containing l vertices, then $\sigma(S) \geqslant \sigma\left(T_{l}\right)$ and, furthermore

$$
\sum_{(i, j) \in S}\left[i\left(1+\frac{1}{n}\right)+j\right] \geqslant \sum_{(i, j) \in T_{i}}\left[i\left(1+\frac{1}{n}\right)+j\right] .
$$

The Corollary will be used in the proof for the three-dimensional grid. And the motivation of the proof is similar to that of the second proof of Theorem 2 .

Let $g_{n}$ be $\{(i, j, k): 1 \leqslant i, j, k \leqslant n\}$ with edges between the vertices that are exactly one unit apart. Let $\nabla$ be the minimum over all indexings of $g_{n}$ of the maximum difference between the integers assigned adjacent points. For all real numbers $a$, let $[a]$ be the greatest integer less than or equal $a$.

TheOrem 3. $\nabla=\left[\frac{3}{4} n^{2}+n / 2\right]$ for $n=2,3, \ldots$

Proof. If $A=(i, j, k)$ is in $g_{n}$, let $O(A)=i(1+1 / n)+j\left(1+1 / n^{2}\right)+k$. Then $O(A)$ is, in a sense, the distance of $A$ to $(1,1,1)$. Note that there is a unique way to index the points of $g_{n}$ so that $O(A)$ increases monotonically as the index of $A$ increases.

For every subset $T$ of $g_{n}$, let $\Sigma(T)=\Sigma_{A \in T} O(A)$.

As in the second proof of Theorem 2, for every subset $T$ of $g_{n}$, let $\sigma(T)$ be the number of points in $g_{n}$ that are not in $T$ but are adjacent to points in $T$. Consider a subset $S$ of $g_{n}$ such that (i) $\sigma(S)$ is minimal among subsets with the same number of points as $S$ and (ii) $\Sigma(S)$ is minimal among sets which satisfy condition (i).

Let $P_{i}=\{(i, j, k)$ : for some integers $j$ and $k,(i, j, k) \in S\}$. Let $P_{i}^{*}$ be $\left\{(i, j, k):(j, k) \in T_{l}\right.$ where $l$ is the number of points in $P_{i}$ and $T_{l}$ is as defined in the Corollary $\}$.

As in the discussion of the previous * operations, $S$ can be compared to $P_{1}^{*} \cup P_{2}^{*} \cup \cdots \cup P_{n}^{*}$. Namely, $\sigma(S) \geqslant \sigma\left(P_{1}^{*} \cup \cdots \cup P_{n}^{*}\right)$. A point $(i, j, k)$ of $g_{n}$, but not of $S$, counts if (i) a point of $S$ having the same $i$ value is adjacent or (ii) either $(i-1, j, k)$ or $(i+1, j, k)$ is in $S$ and (i) does not hold. Clearly, the number of points in each category and thus the number of points in the union is not increased by replacing $S$ by $P_{1}^{*} \cup P_{2}^{*} \cup \cdots \cup P_{n}^{*}$. But if $P_{1}^{*} \cup \cdots \cup P_{n}^{*}$ is not $S$, then $\Sigma$ would be decreased, contradicting the choice of $S$. Hence, $P_{1}^{*} \cup P_{2}^{*} \cup \cdots \cup P_{n}^{*}=S$.

Similarly, for the $j$-direction, $Q_{j}$ slices can be defined and it can be shown $Q_{1}^{*} \cup \cdots \cup Q_{n}^{*}=S$. And, in the $k$-direction, $R_{k}$ slices introduced and $R_{1}^{*} \cup \cdots \cup R_{n}^{*}$ $=S$.

From these facts it follows that if $\left(i_{0}, j_{0}, k_{0}\right)$ is in $S$, then each of the following points that is in $g_{n}$ is in $S:\left(i_{0}, j_{0}-1, k_{0}+1\right),\left(i_{0}-1, j_{0}+1, k_{0}\right)$ and $\left(i_{0}-1, j_{0}, k_{0}\right.$ $+1)$. Let any set $\left\{(i, j, k):(i, j, k) \in g_{n}\right.$ and $\left.i+j+k=c\right\}$ for some constant $c$ be called a face. Then it is easily shown if $\left(i_{0}, j_{0}, k_{0}\right)$ is in $S$ and $\left(i_{1}, j_{1}, k_{1}\right)$ is in the same face and $O\left(i_{0}, j_{0}, k_{0}\right) \geqslant O\left(i_{1}, j_{1}, k_{1}\right)$, then $\left(i_{1}, j_{1}, k_{1}\right)$ is in $S$.

Similarly, if $\left(i_{0}, j_{0}, k_{0}\right) \in S$ and $\left(i_{1}, j_{0}, k_{1}\right) \in g_{n}$ and is in a face corresponding to a smaller constant, then $\left(i_{1}, j_{0}, k_{1}\right) \in S$. It follows that if $\left(i_{0}, j_{0}, k_{0}\right)$ is in $S$, then all but at most $n$ of the points in the face, for which $i+j+k=i_{0}+j_{0}+k_{0}-1$, are in $S_{j}$. Furthermore, if more than $n$ points in the face $i+j+k=i_{0}+j_{0}+k_{0}$ are in $S$, then all the points of the face $i+j+k=i_{0}+j_{0}+k_{0}-1$ are in $S$.

This incomplete description of $S$ suffices for this proof. 


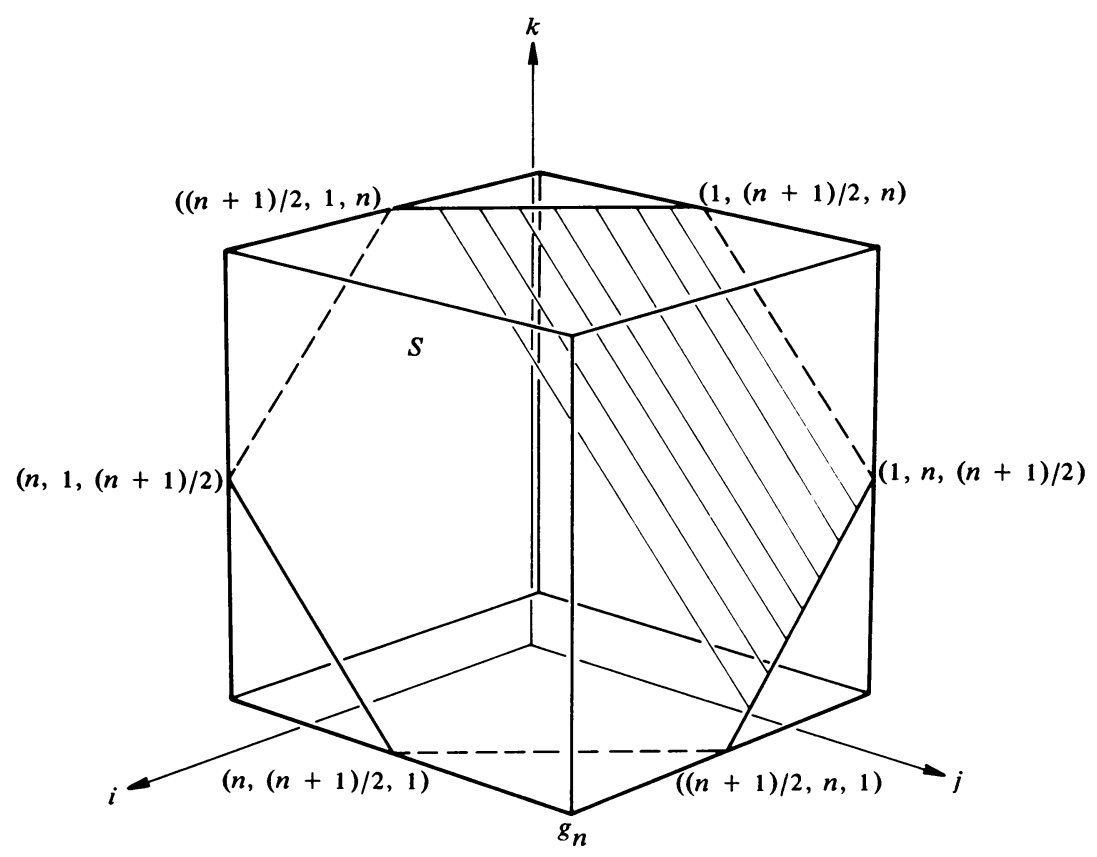

FIGURE 5

Suppose $n$ is odd and $S$ contains $\left(n^{3}-n\right) / 2$ points. The points of $g_{n}$ that are in $S$ are determined by the earlier observations and are illustrated in Fig. 5. They are exactly the points $(i, j, k)$ of $g_{n}$ for which $i+j+k<\left(3 n^{3}+3\right) / 2$ or $i+j+k$ $=\left(3 n^{3}+3\right) / 2$ and $i<(n+1) / 2$. By examining slices perpendicular to the $j$-axis, $\sigma(S)$ can be determined. Among the points of the form $(i, 1, k)$, i.e. in the $Q_{1}$ slice, these points count:

$$
\left(n, 1, \frac{n+1}{2}\right),\left(n-1,1, \frac{n+3}{2}\right), \ldots,\left(\frac{n+1}{2}, 1, n\right), \quad \text { i.e., } \quad \frac{n+1}{2} \text { points. }
$$

In $Q_{2}$, these points count:

$$
\left(n, 2, \frac{n-1}{2}\right), \ldots,\left(\frac{n+1}{2}, 2, n-1\right) \&\left(\frac{n+1}{2}, 2, n\right), \quad \text { i.e., } \frac{n+3}{2} \text { points. }
$$

In $Q_{(n+1) / 2}$, these points count:

$$
\begin{gathered}
\left(n, \frac{n+1}{2}, 1\right), \ldots,\left(\frac{n+1}{2}, \frac{n+1}{2}, \frac{n+1}{2}\right) \&\left(\frac{n+1}{2}, \frac{n+1}{2}, \frac{n+3}{2}\right), \\
\left(\frac{n-1}{2}, \frac{n+1}{2}, \frac{n+5}{2}\right), \ldots,\left(2, \frac{n+1}{2}, n\right) \text { i.e., } n \text { points. }
\end{gathered}
$$

In $Q_{(n+3) / 2}$, these points count:

$$
\begin{aligned}
\left(n-1, \frac{n+3}{2}, 1\right) & \left(n-2, \frac{n+3}{2}, 2\right), \ldots,\left(\frac{n+1}{2}, \frac{n+3}{2}, \frac{n-1}{2}\right) \\
& \&\left(\frac{n+1}{2}, \frac{n+3}{2}, \frac{n+1}{2}\right), \ldots,\left(1, \frac{n+3}{2}, n\right), \text { i.e., } n \text { points. }
\end{aligned}
$$


In $Q_{n}$, these points count:

$$
\begin{aligned}
&\left(\frac{n+1}{2}, n, 1\right) \&\left(\frac{n+1}{2}, n, 2\right),\left(\frac{n-1}{2}, n, 3\right), \ldots,\left(1, n, \frac{n+3}{2}\right) \\
& \text { i.e., } \quad \frac{n+3}{2} \text { points. }
\end{aligned}
$$

Thus

$$
\begin{aligned}
\sigma(S) & =\frac{n+1}{2} \cdot \frac{(n+1) / 2+n}{2}+\frac{n-1}{2} \cdot \frac{n+(n+3) / 2}{2} \\
& =\frac{3}{4} n^{2}+\frac{1}{2} n-\frac{1}{4}=\left[\frac{3}{4} n^{2}+\frac{1}{2} n\right] .
\end{aligned}
$$

An estimate for $\nabla$ follows easily. Given an indexing of $g_{n}$, let $S$ be the set of points with index less than or equal $\left(n^{3}-n\right) / 2$. Since $\sigma(S) \geqslant\left[\frac{3}{4} n^{2}+\frac{1}{2} n\right]$, the point with maximum index among those adjacent to $S$ must have an index differing by at least $\left[\frac{3}{4} n^{2}+\frac{1}{2} n\right]$.

Suppose $n$ is even. If $n$ is 2 , it is clear that $\nabla=\left[\frac{3}{4} n^{2}+\frac{1}{2} n\right]$. If $n$ is greater than 2, let $S$ have $n^{3} / 2-3 n^{2} / 8-3 n / 4$ elements. The subset of $g_{n}$ is determined and has the form depicted in Fig. 5, except the vertices are the points whose coordinates are permutations of $1, \frac{1}{2} n$, and $n$. A similar analysis to the preceding gives $\nabla \geqslant \frac{3}{4} n^{2}+\frac{1}{2} n$ $=\left[\frac{3}{4} n^{2}+\frac{1}{2} n\right]$.

As mentioned at the first of the proof, there is a unique way to index the points of $g_{n}$ from 1 to $n^{3}$ in such a way that $O(A)$ increases monotonically with increasing index. By easy considerations, it is clear that the maximum difference between adjacent indices must occur during the indexing of the middle face or faces of $g_{n}$; and, by counting as in the preceding, this difference is $\left[\frac{3}{4} n^{2}+\frac{1}{2} n\right]$ for this indexing. Hence $\nabla=\left[\frac{3}{4} n^{2}+\frac{1}{2} n\right]$.

Acknowledgements. The author is indebted to Professor A. Garsia, J. Franklin, and Miss $\mathbf{J}$. Revering for discussions of the questions that led to this paper.

Department of Mathematics

University of California, San Diego

La Jolla, California 92037

A. J. Hoffman, M. S. Martin, \& D. J. Rose, "Complexity bounds for regular finite difference and finite element grids," S.I.A.M. J. Numer. Anal., v. 10, 1973, pp. 364-369. 